\title{
Recursive Domain Equations of Filter Models
}

\author{
Fabio Alessi and Paula Severi \\ Università degli Studi di Udine \\ Dipartimento di Matematica ed Informatica \\ via delle Scienze 208, 33100 Udine, Italy
}

\begin{abstract}
Filter models and (solutions of) recursive domain equations are two different ways of constructing lambda models. Many partial results have been shown about the equivalence between these two constructions (in some specific cases). This paper deepens the connection by showing that the equivalence can be shown in a general framework. We will introduce the class of disciplined intersection type theories and its four subclasses: natural split, lazy split, natural equated and lazy equated. We will prove that each class corresponds to a different recursive domain equation. For this result, we are extracting the essence of the specific proofs for the particular cases of intersection type theories and making one general construction that encompasses all of them. This general approach puts together all these results which may appear scattered and sometimes with incomplete proofs in the literature.
\end{abstract}

\section{Introduction}

This paper is concerned with two different ways of constructing models of the untyped lambda calculus which are strongly related.

1. Scott's $D_{\infty}$-models. They were introduced in the 70's as solutions of the recursive domain equation $D=[D \rightarrow D]$ [19]. Their construction depends on an initial domain $D_{0}$ and an initial embedding $i_{0}$. Variations of $D_{0}$ and $i_{0}$ define different $D_{\infty}$-models.

2. Filter models. They were introduced in the 80's using the notion of type as the elementary brick for their construction [5]. We start from an extension of the simply typed lambda calculus with intersection types and subtyping. Then, the interpretation of a $\lambda$-term is defined as the set of its types and it has the property of being a filter. The so-called intersection type theory $\mathcal{T}$ is a set of subtyping statements $A \leq B$. Variations on the intersection type theory $\mathcal{T}$ induce different filter lambda models denoted by $\mathcal{F}^{\mathcal{T}}$.

Many partial results have been shown about the correspondence between both constructions (in some specific cases). Historically, some instances of $D_{0}$ and $i_{0}$ have given rise to some specific filter models such as Scott and Park lambda models $[19,16]$. This result has been generalized $[5,8,9,3]$ by showing that in the category of $\omega$-algebraic complete lattices, any $D_{\infty}$-model can be described 
as a filter model by coding the compact elements of $D_{0}$ as types and defining an intersection type theory $\mathcal{T}$ that contains both the order of $D_{0}$ and $i_{0}{ }^{1}$. The converse is obviously not true. Filter models are in a sense weaker structures than $D_{\infty}$-models. Not all of them satisfy the recursive domain equation $D=[D \rightarrow D]$. If we restrict our attention to the extensional filter models, then some of them have been described as $D_{\infty}$-models by choosing an appropiate $D_{0}$ and $i_{0}[7,8,2,10]$. In spite of the fact that the non-extensional filter models do not satisfy $D=[D \rightarrow D]$, in some cases it is possible to find other recursive domain equations for them [2]. For instance, the non-extensional filter model for the lazy $\lambda$-calculus [1] satisfies the equation $D=[D \rightarrow D]_{\perp}$, while the filter model of [5] satisfies the equation $D=E \times[D \rightarrow D]$ for a suitable $E$. In some cases [8], it is not clear if a filter model satisfy any domain equation at all.

In this paper we show that the connection between filter models and (solutions of) domain equations can be shown in a general framework. We characterize the classes of filter models that correspond to each recursive domain equation as shown in Fig. 1. Many filter models in the literature belong to one of these four classes. Our classification will be done by analyzing patterns of axioms in the definition of the subtyping relation inducing the filter model. In order to give a proper formalization for the study of these patterns of axioms, we will define the subtyping parametric on a set $\Sigma$ of axioms. The judgements are of the form $\Sigma \vdash A \leq B$. This defines the intersection type theory $\mathcal{T}^{\Sigma}$, or just $\mathcal{T}$, generated by $\Sigma$ [8]. Depending on the shape of the axioms $\Sigma$, we will introduce four classes of type theories: natural split, lazy split, natural equated and lazy equated (Section 3). Each class corresponds to a different recursive domain equation in the

\begin{tabular}{|l|l|}
\hline Class & Equation \\
\hline Natural split & $D=E \times[D \rightarrow D]$ \\
Lazy split & $D=E \times[D \rightarrow D]_{\perp}$ \\
Natural equated & $D=[D \rightarrow D]$ \\
Lazy equated & $D=[D \rightarrow D]_{\perp}$ \\
\hline
\end{tabular}

Fig. 1. Classification of recursive domain equations

following sense: the intersection type theory $\mathcal{T}$ belongs to the class iff the filter model $\mathcal{F}^{\mathcal{T}}$ satisfies the corresponding recursive domain equation. This result will be a consequence of a stronger and more refined theorem which states the correspondence between intersection type theories and triples $\left\langle F, D_{0}, i_{0}\right\rangle$ where $F$ is a functor, $D_{0}$ an object and $i_{0}$ an embedding in the category of complete $\omega$-algebraic lattices. We will specify in each of the four cases how to construct

\footnotetext{
${ }^{1}$ In the categories of Scott domains or stable sets, $D_{\infty}$-models cannot be captured in their full generality.
} 
$\mathcal{F}^{\mathcal{T}}$ as a colimit of $\omega$-chains starting from certain $\left\langle F, D_{0}, i_{0}\right\rangle$ (Section 4 ).

The contribution of this paper can be summarized as follows.

1. We introduce the class of disciplined type theories and its four subclasses: natural split, lazy split, natural equated and lazy equated.

2. In Theorem 27, we prove that filter models over any disciplined intersection type theory can be constructed as the colimit of certain $\omega$-chain. In Theorem 30 , we prove the converse.

3. As corollaries of those two theorems, we deduce the correspondence between intersection type theories and recursive domain equations shown in Fig. 1

4. In the proofs of Theorem 27 and Theorem 30, we are extracting the essence of the specific proofs for Scott, Park, CDZ, DHM and HR and making one general construction that encompasses all these particular cases. This general approach puts together all these results which may appear scattered and sometimes with incomplete proofs in the literature.

\section{Domain-theoretic Preliminaries}

This auxiliary section is devoted to recall some definition and facts from lattice theory of some importance in the following sections. For fundamental notions and results on lattices we refer to [13].

Definition 1. 1. Let $D=\langle D, \sqsubseteq\rangle$ be a complete lattice. A subset $Z \subseteq D$ is directed if it is non-empty and for any $z, z^{\prime} \in Z$ there exists $z^{\prime \prime} \in Z$ such that $z, z^{\prime} \sqsubseteq z^{\prime \prime}$.

2. A monotone function $f: D \rightarrow E$ is continuous if for any directed $Z \subseteq D$, we have that $f(\bigsqcup Z)=\bigsqcup f(Z)$. The space of continuous functions from $D$ to $E$, ordered with the pointwise ordering, is denoted by $[D \rightarrow E]$.

3. An element $d \in D$ is compact if for any directed $Z \subseteq D, d \sqsubseteq \bigsqcup Z$ implies that there exists $z \in Z$ such that $d \sqsubseteq z$. The set of compact elements of $D$ is denoted by $\mathcal{K}(D)$.

4. $D$ is an $\omega$-algebraic lattice if $\mathcal{K}(D)$ is countable and moreover, for any $x \in D$, $x=\bigsqcup\{d \in \mathcal{K}(D) \mid d \sqsubseteq x\}$. ALG is the category of complete $\omega$-algebraic lattices and continuous functions (see [17]).

5. Given two $\omega$-algebraic lattices $D$ and $E$, and two compact elements $d \in$ $\mathcal{K}(D), e \in \mathcal{K}(E)$, we define the step function

$$
(d \Rightarrow e)(x)=\left\{\begin{array}{l}
e \text { if } d \sqsubseteq x \\
\perp \text { otherwise }
\end{array}\right.
$$

We have that $(d \Rightarrow e) \sqsubseteq f$ if and only if $e \sqsubseteq f(d)$. Hence $d \Rightarrow e \sqsubseteq d^{\prime} \Rightarrow e^{\prime}$ if and only if $d^{\prime} \sqsubseteq d$ and $e \sqsubseteq e^{\prime}$. Finite sups of step functions are the compact elements in $[D \rightarrow E]$.

Lemma 2. Let $D, E \in$ ALG. Then,

$$
\text { 1. } \mathcal{K}([D \rightarrow E])=\left\{\bigsqcup_{i=1}^{n}\left(d_{i} \Rightarrow e_{i}\right) \mid n \in N \& d_{i}, e_{i} \text { compact \& } 1 \leq i \leq n\right\} \text {. }
$$


2. $[D \rightarrow E]$ is $\omega$-algebraic.

3. $\bigsqcup_{j=1}^{n}\left(c_{j} \Rightarrow d_{j}\right) \sqsubseteq \bigsqcup_{i=1}^{n}\left(a_{i} \Rightarrow b_{i}\right) \Leftrightarrow$

$\forall j \in\{1 \ldots n\} . d_{j} \sqsubseteq \bigsqcup\left\{b_{i} \mid 1 \leq i \leq n \& a_{i} \sqsubseteq c_{j}\right\}$.

Morever ALG is a CCC (cartesian closed category) with "enough points".

Definition 3. Let $i: D \rightarrow E, j: E \rightarrow D$ be continuous functions. We say that $\iota=\langle i, j\rangle: D \rightarrow E$ is an embedding-projection pair (ep for short) if $j \circ i=I d_{D}$ and $i \circ j \sqsubseteq I d_{E}$.

If $\langle i, j\rangle: D \rightarrow E$ and $\langle h, k\rangle: E \rightarrow E^{\prime}$, then $\langle i, j\rangle \circ\langle h, k\rangle=\langle h \circ i, j \circ k\rangle$.

$\mathbf{A L G}^{E}$ is the category of $\omega$-algebraic lattices and $e p$ 's.

Next lemma on ep's is very useful. Its proof can be recovered by using the results of Section 0-3 of [13] on basic properties of Galois connections.

Lemma 4. Let $D, E \in \mathbf{A L G}$ and $\iota=\langle i, j\rangle: D \rightarrow E$ be an ep.

1. $\forall x \in D, y \in E . i(x) \sqsubseteq y \Leftrightarrow x \sqsubseteq j(y)$.

$j$ is the right adjoint of $i$ and it is often denoted by $i^{R}$.

2. $\iota$ is completely determined by the embedding $i$, since $j$ is forced to satisfy the following equality:

$$
(\dagger) j(y)=\bigsqcup\{x \mid i(x) \sqsubseteq y\}
$$

3. $i$ is additive, injective and preserves compact elements.

Thanks to Lemma 4(2), we identify an ep $\iota=\left\langle i, i^{R}\right\rangle$ with its embedding $i$.

Definition 5. Let $F: \mathbf{A L G}{ }^{E} \rightarrow \mathbf{A L G}^{E}$ be a locally continuous endofunctor ${ }^{2}$, $D_{0} \in \mathbf{A L G}$ and $i_{0}: D_{0} \rightarrow F\left(D_{0}\right)$ be an embedding in ALG.

1. The triple $\rho=\left\langle F, D_{0}, i_{0}\right\rangle$ is called a specification of a colimit.

2. Every specification $\rho=\left(F, D_{0}, i_{0}\right)$, induces an $\omega$-chain

$$
D_{0} \stackrel{i_{0}}{\rightarrow} F\left(D_{0}\right) \stackrel{F\left(i_{0}\right)}{\rightarrow} F^{2}\left(D_{0}\right) \stackrel{F^{2}\left(i_{0}\right)}{\rightarrow} F^{3}\left(D_{0}\right) \ldots
$$

that we call the $\omega$-chain of $\rho$.

3. Then, $D_{0}$ is called the initial domain and $i_{0}$ the initial embedding.

4. The colimit of $\rho$, denoted by $\operatorname{colim}(\rho)$, is the colimit of the $\omega$-chain of $\rho$.

\section{Intersection Type Theories and Filter Models}

In this section we recall the notion of intersection type theory and its induced filter model. We will consider intersection type theories generated by a set $\Sigma$ of axioms [8]. Depending on the shape of the axioms $\Sigma$, we introduce four classes of type theories: natural split, lazy split, natural equated and lazy equated. We show that many of the examples of intersection type theories that appear in the literature fit in one of these four patterns of axioms. In this section we will also show that any of these four classes behaves well in the sense that they all induce reflexive filter structures and, hence, $\lambda$-models.

${ }^{2}$ See [20]: what matters here is that $F$ locally continuous implies that it commutes with colimits in $\mathbf{A} \mathbf{L} \mathbf{G}^{E}$ and the domain equation $X=F(X)$ has solution in ALG. 
Definition 6. Let $\mathbb{A}$ be a countable set of symbols, called atoms.

1. We assume there is a special atom $\top$ in $\mathbb{A}$, called top.

2. The set $\mathbb{B}(\mathbb{A})$ (or just $\mathbb{B}$ ) of basic types over $\mathbb{A}$ is defined by $\mathbb{B}=\mathbb{A} \mid \mathbb{B} \cap \mathbb{B}$.

3. The set $\mathbb{T}(\mathbb{A})$ (or just $\mathbb{T}$ ) of types over $\mathbb{A}$ is defined by $\mathbb{T}=\mathbb{A}|\mathbb{T} \rightarrow \mathbb{T}| \mathbb{T} \cap \mathbb{T}$.

Greek letters $\alpha, \beta, \ldots$ range over $\mathbb{A}$ and $A, B, \ldots$ range over $\mathbb{B}$ or $\mathbb{T}$.

Next definition is standard [5] except for the axiom ( $\left.T_{\text {lazy }}\right)$. The intuition behind this last axiom is that anything that is more defined than a function is still a function. Note that we can deduce that $A \rightarrow \top=\top \rightarrow T$.

Definition 7. $A n$ intersection type theory $\mathcal{T}$ is a set of statements of the form $A \leq B$ (to be read: $A$ is a subtype of $B$ ), with $A, B \in \mathbb{T}$, that satisfies the following axioms and rules.

\begin{tabular}{|c|c|c|c|}
\hline (refl) $\quad A \leq A$ & (trans) & $\frac{A \leq B \quad B \leq C}{A \leq C}$ & ( $\top) \quad A \leq \top$ \\
\hline $\begin{array}{l}\left(\operatorname{incl}_{L}\right) A \cap B \leq A \\
\left(\operatorname{incl}_{R}\right) A \cap B \leq B\end{array}$ & (glb) & $\frac{C \leq A \quad C \leq B}{C \leq A \cap B}$ & $\left(\top_{\text {lazy }}\right) A \rightarrow \top \leq \top \rightarrow \top$ \\
\hline ( ) ( & & $\rightarrow B \cap C$ & $(\rightarrow) \frac{A^{\prime} \leq A \quad B \leq B^{\prime}}{A \rightarrow B \leq A^{\prime} \rightarrow B^{\prime}}$ \\
\hline
\end{tabular}

We write $A=B$ (to be read as " $A$ is equivalent to $B$ ") for $A \leq B \in \mathcal{T}$ and $B \leq A \in \mathcal{T}$. We extend $\leq, \cap$ and $\rightarrow$ to $\mathbb{T} /=$ in the obvious way. Syntactic identity is denoted as $A \equiv B$.

Intersection is associative and commutative with respect to equivalence of types, so if $n \geq 1$, we may write $\bigcap_{i=1}^{n} A_{i}$ or $\bigcap\left\{A_{1}, \cdots, A_{n}\right\}$ for $A_{1} \cap \cdots \cap A_{n}$. The case $\bigcap \emptyset$ denotes $T$. In the literature, the top $T$ is denoted by different symbols such as $\omega$ in [6] or $\Omega$ in [11]. If $n \geq 1$, we may write $\bigcap_{i=1}^{n} A_{i}$ or $\bigcap\left\{A_{1}, \cdots, A_{n}\right\}$ for $A_{1} \cap \cdots \cap A_{n}$. The case $\bigcap \emptyset$ denotes $\top$. In the literature, the top $\top$ is denoted by different symbols such as $\omega$ in [6] or $\Omega$ in [11].

We now recall the definition of filter structure $[5,8]$.

Definition 8. Let $\mathcal{T}$ be an intersection type theory.

1. A filter (resp. basic filter) is a set $X \subseteq \mathbb{T}$ (resp. $X \subseteq \mathbb{B}$ ) such that:

(a) $T \in X$;

(b) $A \leq B\left(\right.$ resp. $\left.A \leq_{\mathcal{B}} B\right)$ and $A \in X$ imply $B \in X$;

(c) $A \in X$ and $B \in X$ imply $A \cap B \in X$.

2. Let $Y \subseteq \mathbb{T}$. Then, $\uparrow Y$ denotes the filter generated by $Y$. If $Y=\{A\}$, we write $\uparrow A$ instead of $\uparrow\{A\}$ and $\uparrow A$ is called a principal filter. Actually it coincides with the upper closure of $A$, i.e. $\uparrow A=\{B \mid A \leq B\}$. If $Y \subseteq \mathbb{B}$ the basic filter generated by $Y$ is denoted by $\uparrow^{\mathcal{B}} Y$. Similarly, we write $\uparrow^{\mathcal{B}} A$ instead of $\uparrow^{\mathcal{B}}\{A\}$ for the principal filter.

3. $\mathcal{F}^{\mathcal{T}}$ (resp. $\mathcal{F}^{\mathcal{B}}$ ) is the set of filters (resp. basic filters) over $\mathcal{T}$, ordered by set-theoretic inclusion, and is called the filter structure (resp. basic filter structure) over $\mathcal{T}$. 
It is well-known that $\mathcal{F}^{\mathcal{T}}$ is an $\omega$-algebraic lattice [8]. Given $\mathcal{X} \subseteq \mathcal{F}^{\mathcal{T}}$

$$
\text { (fil-sup) } \bigsqcup \mathcal{X}=\uparrow\left\{\bigcap_{j=1}^{n} A_{j} \mid n \in \mathbb{N}, \forall 1 \leq j \leq n . \exists X \in \mathcal{X} . A_{j} \in X\right\},
$$

Moreover $X \sqcap Y=X \cap Y$, the bottom filter is $\uparrow \top$, the top filter is $\mathbb{T}$. Compact elements in $\mathcal{F}^{\mathcal{T}}$ are the principal filters and they inherit the order $\leq^{o p}$ :

$$
\uparrow A \subseteq \uparrow B \Leftrightarrow B \leq A
$$

In the following definition we show how to interpret the untyped lambda calculus in a filter structure $\mathcal{F}^{\mathcal{T}}$.

Definition 9. Let $\mathcal{T}$ be an intersection type theory.

1. We define $\operatorname{App}^{\mathcal{T}}: \mathcal{F}^{\mathcal{T}} \rightarrow\left[\mathcal{F}^{\mathcal{T}} \rightarrow \mathcal{F}^{\mathcal{T}}\right]$ and $\mathrm{Abs}^{\mathcal{T}}:\left[\mathcal{F}^{\mathcal{T}} \rightarrow \mathcal{F}^{\mathcal{T}}\right] \rightarrow \mathcal{F}^{\mathcal{T}}$ as follows.

$$
\begin{aligned}
& \operatorname{App}^{\mathcal{T}}(X)(Y)=\{B \mid \exists A \in Y . A \rightarrow B \in X\} \\
& \operatorname{Abs}^{\mathcal{T}}(f)=\uparrow\{A \rightarrow B \mid B \in f(\uparrow A)\}
\end{aligned}
$$

2. We define an interpretation on $\lambda$-terms as follows $[8]^{3}$.

$$
\begin{aligned}
& \llbracket x \rrbracket_{\rho}=\rho(x) \\
& \llbracket M N \rrbracket_{\rho}=\left(\operatorname{App}^{\mathcal{T}}\left(\llbracket M \rrbracket_{\rho}\right)\right)\left(\llbracket N \rrbracket_{\rho}\right) \\
& \llbracket \lambda x \cdot M \rrbracket_{\rho}=\operatorname{Abs}^{\mathcal{T}}\left(\boldsymbol{\lambda} d \in \mathcal{F}^{\mathcal{T}} \cdot \llbracket M \rrbracket_{\rho[x / d]}\right)
\end{aligned}
$$

Not any filter structure $\mathcal{F}^{\mathcal{T}}$ gives rise to a lambda model $\left\langle\mathcal{F}^{\mathcal{T}}, \mathrm{App}^{\mathcal{T}}, \mathrm{Abs}^{\mathcal{T}}, \llbracket \llbracket \rrbracket\right\rangle$, but if the definition of an intersection type theory satisfy some restrictions, not only the induced filter structure will be a $\lambda$-model, but also the connection with suitable colimits emerges rather clearly. Importantly, the restrictions we put in Definition 12 are easily satisfied by the main intersection type theories in the literature. Before that we have to introduce the notion of specification of axioms.

Definition 10. A specification of axioms is a pair $\Sigma=\left(<_{\mathcal{B}}\right.$, def $)$ where $<_{\mathcal{B}}$ is a partial order on $\mathbb{B}$ such that $\cap$ is the meet and $T$ is the top and def is a function from $\mathbb{A}$ to $\mathbb{T}$. The function def is extended from $\mathbb{A}$ to $\mathbb{T}$ by $\operatorname{def}(A \cap B)=$ $\operatorname{def}(A) \cap \operatorname{def}(B)$ and $\operatorname{def}(A \rightarrow B)=A \rightarrow B$.

Definition 11. Let $\Sigma=\left(<_{\mathcal{B}}\right.$, def $)$ be a specification of axioms. The intersection type theory generated by $\Sigma$, denoted by $\mathcal{T}^{\Sigma}$ or just $\mathcal{T}$, derives judgements of the form $A \leq B \in \mathcal{T}^{\Sigma}$ or $\Sigma \vdash A \leq B$ and it is defined as the smallest intersection type theory that contains the following two axioms.

$$
(\mathcal{B} \text {-ax }) \frac{A \leq_{\mathcal{B}} B}{\Sigma \vdash A \leq B} \quad(\text { def-ax }) \Sigma \vdash \alpha=\operatorname{def}(\alpha)
$$

We write $\Sigma \vdash A \leq B$ if $A \leq B \in \mathcal{T}^{\Sigma}$. If there is little danger of confusion, when $\Sigma$ or $\mathcal{T}$ are clear from the context, then we will just write $(A \leq B)$.

Figure 2 shows how many of the intersection type theories that appear in the literature can be generated by a specification $\Sigma=\left(\leq_{\mathcal{B}}\right.$, def $)$. For the $\mathcal{B}$-axioms, 


\begin{tabular}{|c|c|c|c|}
\hline $\mathcal{T}$ & $\mathbb{A}$ & $\mathcal{B}$-axioms $\leq_{\mathcal{B}}$ & def-axioms $\quad \alpha=\operatorname{def}(\alpha)$ \\
\hline Scott [19] & $\{0, \top\}$ & $0 \leq_{\mathcal{B}} \top$ & $0=\top \rightarrow 0, T=T \rightarrow \top$ \\
\hline Park [16] & $\{0, \top\}$ & $0 \leq_{\mathcal{B}} \top$ & $0=0 \rightarrow 0, T=T \rightarrow T$ \\
\hline CDZ [9] & $\{0,1, \top\}$ & $0 \leq_{\mathcal{B}} 1 \leq_{\mathcal{B}} \top$ & $0=1 \rightarrow 0,1=0 \rightarrow 1, \top=\top \rightarrow \top$ \\
\hline HR [15] & $\{0,1, \top\}$ & $0 \leq_{\mathcal{B}} 1 \leq_{\mathcal{B}} \top$ & $0=1 \rightarrow 0,1=(0 \rightarrow 0) \cap(1 \rightarrow 1), \top=\top \rightarrow \top$ \\
\hline DHM $[12]$ & $\{0,1, \top\}$ & $0 \leq_{\mathcal{B}} 1 \leq_{\mathcal{B}} \top$ & $0=\top \rightarrow 0,1=0 \rightarrow 1, \top=\top \rightarrow \top$ \\
\hline BCD [5] & $\mathbb{A}^{\infty}$ & $\mathrm{c}_{i} \leq_{\mathcal{B}} \top$ & $\mathrm{c}_{i}=\mathrm{c}_{i}, \top=\top \rightarrow \top$ \\
\hline $\mathrm{AO}[1]$ & $\{T\}$ & & $T=T$ \\
\hline
\end{tabular}

Fig. 2. Intersection type theories generated by $\Sigma=\left(\leq_{\mathcal{B}}\right.$, def $)$

we do not specify the whole set $\leq_{\mathcal{B}}$. Each $\leq_{\mathcal{B}}$ is actually defined as the least partial order that contains the pairs $A \leq_{\mathcal{B}} B$ shown in the table. We define a countable set of constants $\mathbb{A}^{\infty}=\left\{\mathrm{c}_{i} \mid i \in N\right\} \cup\{\top\}$.

Definition 12. Let $\Sigma=\left(<_{\mathcal{B}}\right.$, def $)$ be a specification of axioms.

1. We say that $\Sigma$ (and also $\mathcal{T}^{\Sigma}$ ) is lazy if $\operatorname{def}(\top)=\top$.

2. We say that $\Sigma$ (and also $\mathcal{T}^{\Sigma}$ ) is natural if $\operatorname{def}(\top)=\top \rightarrow \top$.

3. We say that $\Sigma$ (and also $\mathcal{T}^{\Sigma}$ ) is split if the following two conditions hold.

(a) $\mathbb{A}-\{\top\} \neq \emptyset$.

(b) $\operatorname{def}(\alpha)=\alpha, \forall \alpha \in \mathbb{A}-\{\top\}$.

4. We say that $\Sigma$ (and also $\mathcal{T}^{\Sigma}$ ) is equated if the following conditions hold.

(a) $\forall \alpha \in \mathbb{A}-\{\top\}, \exists A_{1}, \ldots A_{n} \in \mathbb{B}, B_{1}, \ldots B_{n} \in \mathbb{B}-\{\top\}$,

$$
\operatorname{def}(\alpha) \equiv \bigcap_{i=1}^{n}\left(A_{i} \rightarrow B_{i}\right)
$$

(b) $\forall A, B \in \mathbb{B}-\{\top\}$,

$$
A \leq_{\mathcal{B}} B \Leftrightarrow \forall j \in\{1 \ldots n\} . \bigcap\left\{B_{i} \mid 1 \leq i \leq m \& A_{i} \geq_{\mathcal{B}} C_{j}\right\} \leq_{\mathcal{B}} D_{j}
$$

where $\operatorname{def}(A) \equiv \bigcap_{i=1}^{m}\left(A_{i} \rightarrow B_{i}\right)$ and $\operatorname{def}(B) \equiv \bigcap_{j=1}^{n}\left(C_{j} \rightarrow D_{j}\right)$.

5. We say that $\Sigma$ (and also $\mathcal{T}^{\Sigma}$ ) is disciplined if it is either one of these four possible combinations: natural split, lazy split, natural equated or lazy equated.

The examples of intersection type theories given in Figure 2 are classified according to the four classes defined above as follows.

$\begin{array}{ll}\text { Natural split } & \text { BCD } \\ \text { Lazy split } & \text { none } \\ \text { Natural equated } & \text { Scott, Park, CDZ, HR, DHM } \\ \text { Lazy equated } & \text { AO }\end{array}$

${ }^{3}$ It coincides with the interpretation defined through the type assignment system [8]. 
Lemma 13. In any intersection type theory $\mathcal{T}$, the following statements hold:

1. If $A \leq C$ and $B \leq D$ then $A \cap B \leq C \cap D$.

2. $\left(A_{1} \rightarrow B_{1}\right) \cap \ldots \cap\left(A_{n} \rightarrow B_{n}\right) \leq\left(A_{1} \cap \ldots \cap A_{n}\right) \rightarrow\left(B_{1} \cap \ldots \cap B_{n}\right)$.

3. If $\forall j \in\{1 \ldots n\} . \bigcap\left\{B_{i} \mid 1 \leq i \leq m \& A_{i} \geq C_{j}\right\} \leq D_{j}$ then

$$
\bigcap_{i=1}^{m}\left(A_{i} \rightarrow B_{i}\right) \leq \bigcap_{j=1}^{n}\left(C_{j} \rightarrow D_{j}\right) .
$$

Definition 14. We define two functions to extract the sets of outermost atoms and arrows of a type.

$$
\begin{array}{ll}
\operatorname{ats}(\alpha)=\{\alpha\} & \operatorname{ars}(\alpha)=\emptyset \\
\operatorname{ats}(A \rightarrow B)=\emptyset & \operatorname{ars}(A \rightarrow B)=\{A \rightarrow B\} \\
\operatorname{ats}(A \cap B)=\operatorname{ats}(A) \cup \operatorname{ats}(B) & \operatorname{ars}(A \cap B)=\operatorname{ars}(A) \cup \operatorname{ars}(B)
\end{array}
$$

Theorem 15. (Conservativity of $\leq_{\mathcal{B}}$ ). Let $A, B \in \mathbb{B}$ and $\Sigma$ be disciplined. Then, $A \leq_{\mathcal{B}} B$ iff $\Sigma \vdash A \leq B$.

Since $\leq_{\mathcal{B}}$ is antisymmetric, we have the following:

Corollary 16. If $\Sigma \vdash \alpha=\beta$ then $\alpha \equiv \beta$.

The converse of Lemma 13 part 3 is an important property which is not always true. We will see later that it is a sufficient and necesary condition for having a reflexive filter structure. For this, we will define the following notion.

Definition 17. We say that $\mathcal{T}$ is $\beta$-sound if

$$
\bigcap_{i=1}^{m}\left(A_{i} \rightarrow B_{i}\right) \leq(C \rightarrow D) \Rightarrow \bigcap\left\{B_{i} \mid 1 \leq i \leq m \& A_{i} \geq C\right\} \leq D
$$

$\forall A_{i}, B_{i}, C, D \in \mathbb{T}$ with $1 \leq i \leq m$.

A particular case of $\beta$-soundness is the invertibility of the rule $(\rightarrow)$, i.e. if $A \rightarrow$ $B \leq C \rightarrow D$ then $A \geq C$ and $B \leq D$.

Theorem 18. Let $\mathcal{T}$ be disciplined. If $E \leq F$ then

1. $\bigcap$ ats $(\operatorname{def}(E)) \leq \bigcap$ ats $(\operatorname{def}(F))$

2. $\forall j \in\{1 \ldots n\} . \bigcap\left\{B_{i} \mid 1 \leq i \leq m \& A_{i} \geq C_{j}\right\} \leq D_{j}$ where $\operatorname{ars}(\operatorname{def}(E)) \equiv\left\{\left(A_{i} \rightarrow B_{i}\right) \mid 1 \leq i \leq m\right\}$ and $\operatorname{ars}(\operatorname{def}(F)) \equiv\left\{\left(C_{j} \rightarrow D_{j}\right) \mid 1 \leq i \leq n\right\}$.

Proof. We prove it by induction on the derivation of $E \leq F$. The interesting case is $(\mathcal{B}$-ax) which follows from Condition $4 \mathrm{~b}(\Rightarrow)$ in Definition 12.

Corollary 19. Let $\mathcal{T}$ be disciplined. Then $\mathcal{T}$ is $\beta$-sound.

Theorem 20. If $\mathcal{T}$ is disciplined then $\left\langle\mathcal{F}^{\mathcal{T}}, \mathrm{App}^{\mathcal{T}}, \mathrm{Abs}^{\mathcal{T}}\right.$, $\left.\llbracket \rrbracket\right\rangle$ is a $\lambda$-model.

Proof. Since $\mathcal{T}$ is $\beta$-sound, it follows from $[8,18]$ that $\left\langle\mathcal{F}^{\mathcal{T}}, \mathrm{App}^{\mathcal{T}}, \mathrm{Abs}^{\mathcal{T}}, \llbracket-\rrbracket\right\rangle$ is a reflexive filter structure, i.e. $\mathrm{App}^{\mathcal{T}} \circ \mathrm{Abs}^{\mathcal{T}}=I d_{\left[\mathcal{F}^{\mathcal{T}} \rightarrow \mathcal{F}^{\mathcal{T}}\right]}$. Since any reflexive filter structure is a $\lambda$-model $[14,4]$, we conclude that $\mathcal{F}^{\mathcal{T}}$ is also a $\lambda$-model. 


\section{Classification of Recursive Domain Equations}

This section shows that filter structures over disciplined type theories correspond to the colimit of certain $\omega$-chains. Definition 24 gives the correspondence between the intersection type theories $\mathcal{T}$ and the specification $\rho^{(\mathcal{T})}=\left\langle F^{(\mathcal{T})}, D_{0}^{(\mathcal{T})}, i_{0}^{(\mathcal{T})}\right\rangle$ of a colimit. In Theorem 27, we prove that $\mathcal{F}^{\mathcal{T}}$ is isomorphic to the colimit of $\left(F^{(\mathcal{T})}, D_{0}^{(\mathcal{T})}, i_{0}^{(\mathcal{T})}\right)$. In Theorem 30 we prove the converse: for any triple $\left(F, D_{0}, i_{0}\right)$ of certain class $\mathcal{C}$, it is possible to construct a displined $\mathcal{T}$ such that $\mathcal{F}^{\mathcal{T}}$ is isomorphic to the colimit of $\left(F, D_{0}, i_{0}\right)$. As a consequence of these two theorems, we can justify the correspondence between classes and recursive domain equations shown in Figure 1.

Definition 21. We define four different functors in $\mathbf{A} \mathbf{L G}{ }^{E}$.

$$
\begin{aligned}
& \operatorname{Hom}^{Y}(X)=Y \times[X \rightarrow X] \\
& \operatorname{Hom}_{\perp}^{Y}(X)=Y \times[X \rightarrow X]_{\perp} \\
& \operatorname{Hom}(X)=[X \rightarrow X] \\
& \operatorname{Hom}_{\perp}(X)=[X \rightarrow X]_{\perp}
\end{aligned}
$$

with the expected actions on morphisms (for instance, $\operatorname{Hom}(i)(f)=i \circ f \circ j$ for an embedding projection pair $\langle i, j\rangle$ and $f: X \rightarrow X$ a continous function).

Definition 22. Let $D_{0}=\{\perp\}$. The trivial embedding trv $: D_{0} \rightarrow F\left(D_{0}\right)$ is defined as $\operatorname{trv}(\perp)=\perp$.

Definition 23. Let $\Sigma$ be equated. We define the embedding def : $\mathcal{F}^{\mathcal{B}} \rightarrow\left[\mathcal{F}^{\mathcal{B}} \rightarrow\right.$ $\mathcal{F}^{\mathcal{B}}$ on compact elements as follows.

1. If $\operatorname{def}(A) \equiv \bigcap_{i=1}^{m}\left(A_{i} \rightarrow B_{i}\right)$ then

$$
\begin{aligned}
& \hat{\operatorname{def}}\left(\uparrow^{\mathcal{B}} A\right)= \begin{cases}\bigsqcup_{i=1}^{m}\left(\uparrow^{\mathcal{B}} A_{i} \Rightarrow \uparrow^{\mathcal{B}} B_{i}\right) & \text { if } \Sigma \text { is natural } \\
\left(\bigsqcup_{i=1}^{m}\left(\uparrow^{\mathcal{B}} A_{i} \Rightarrow \uparrow^{\mathcal{B}} B_{i}\right), 0\right) & \text { if } \Sigma \text { is lazy }\end{cases} \\
& \text { 2. If } \operatorname{def}(\top)=\top \text { then } \operatorname{def}\left(\uparrow^{\mathcal{B}} \top\right)=\perp .
\end{aligned}
$$

Definition 24. Let $\mathcal{T}$ be disciplined. We define a specification of a colimit according to the following four cases.

\begin{tabular}{|l|l|l|l|}
\hline $\mathcal{T}$ & $F^{(\mathcal{T})}$ & $D_{0}^{(\mathcal{T})}$ & $i_{0}^{(\mathcal{T})}$ \\
\hline Natural split & $\operatorname{Hom}^{\mathcal{F}^{\mathcal{B}}}$ & $\{\perp\}$ & $\operatorname{trv}$ \\
Lazy split & $\operatorname{Hom}_{\perp}^{\mathcal{F}^{\mathcal{B}}}$ & $\{\perp\}$ & $\operatorname{trv}$ \\
Natural equated & $\mathrm{Hom}^{\mathcal{B}}$ & $\mathcal{F}^{\mathcal{B}}$ & def \\
Lazy equated & $\operatorname{Hom}_{\perp}$ & $\mathcal{F}^{\mathcal{B}}$ & def \\
\hline
\end{tabular}

Finally, for any $n>0$, define inductively $D_{n}^{(\mathcal{T})}=F^{(\mathcal{T})}\left(D_{n-1}\right)$. 
Definition 25. Let $\mathcal{T}$ be disciplined. We define $\mu_{n}^{(\mathcal{T})}: \mathcal{K}\left(D_{n}^{(\mathcal{T})}\right) \rightarrow \mathbb{T} /=$ by induction as follows.

$$
\begin{aligned}
& \text { 1. - if } \mathcal{T} \text { is split, then } \mu_{0}^{(\mathcal{T})}(\perp)=[\mathrm{T}] \\
& - \text { if } \mathcal{T} \text { is equated, then } \mu_{0}^{(\mathcal{T})}\left(\uparrow^{\mathcal{B}} A\right)=[A] \\
& \text { 2. Let } n>0 \text {. Then } \\
& - \text { if } \mathcal{T} \text { is natural split, then } \\
& \quad \mu_{n}^{(\mathcal{T})}\left(\uparrow^{\mathcal{B}} A, \bigsqcup_{i=1}^{m}\left(d_{i} \Rightarrow e_{i}\right)\right)=[A] \cap\left(\bigcap_{i=1}^{m}\left(\mu_{n-1}^{(\mathcal{T})}\left(d_{i}\right) \rightarrow \mu_{n-1}^{(\mathcal{T})}\left(e_{i}\right)\right)\right. \\
& - \text { if } \mathcal{T} \text { is lazy split, then } \\
& \quad \mu_{n}^{(\mathcal{T})}\left(\uparrow^{\mathcal{B}} A, \perp\right)=[A] \\
& \quad \mu_{n}^{(\mathcal{T})}\left(\uparrow^{\mathcal{B}} A,\left(\bigsqcup_{i=1}^{m}\left(d_{i} \Rightarrow e_{i}\right), 0\right)\right)=[A] \cap\left(\bigcap_{i=1}^{m}\left(\mu_{n-1}^{(\mathcal{T})}\left(d_{i}\right) \rightarrow \mu_{n-1}^{(\mathcal{T})}\left(e_{i}\right)\right)\right. \\
& - \text { if } \mathcal{T} \text { is natural equated, then } \\
& \mu_{n}^{(\mathcal{T})}\left(\bigsqcup_{i=1}^{m}\left(d_{i} \Rightarrow e_{i}\right)\right)=\bigcap_{i=1}^{m}\left(\mu_{n-1}^{(\mathcal{T})}\left(d_{i}\right) \rightarrow \mu_{n-1}^{(\mathcal{T})}\left(e_{i}\right)\right) \\
& - \text { if } \mathcal{T} \text { is lazy equated, then } \\
& \quad \mu_{n}^{(\mathcal{T})}(\perp)=[\mathcal{T}] \\
& \quad \mu_{n}^{(\mathcal{T})}\left(\bigsqcup_{i=1}^{m}\left(d_{i} \Rightarrow e_{i}\right), 0\right)=\bigcap_{i=1}^{m}\left(\mu_{n-1}^{(\mathcal{T})}\left(d_{i}\right) \rightarrow \mu_{n-1}^{(\mathcal{T})}\left(e_{i}\right)\right) .
\end{aligned}
$$

From now on we omit the superscript $(\mathcal{T})$ on $\mu_{n}$.

Proposition 26. Let $\mathcal{T}$ be disciplined. Then for all $n$,

1. $\forall e, e^{\prime} \in \mathcal{K}\left(D_{n}^{(\mathcal{T})}\right) \cdot e^{\prime} \sqsubseteq e \Leftrightarrow \mu_{n}(e) \leq \mu_{n}\left(e^{\prime}\right)$.

2. $\forall d \in \mathcal{K}\left(D_{n}^{(\mathcal{T})}\right) \cdot \mu_{n}(d)=\mu_{n+1}\left(i_{n}(d)\right)$ where $i_{n}=F^{(\mathcal{T})}{ }^{(n)}\left(i_{0}\right)$.

3. $\forall A \in \mathbb{T} . \exists n \geq 0, a \in \mathcal{K}\left(D_{n}^{(\mathcal{T})}\right) . \mu_{n}(a)=[A]$.

Theorem 27. Let $\mathcal{T}$ be disciplined. Then

$$
\mathcal{F}^{\mathcal{T}} \simeq \operatorname{colim}\left(\rho^{(\mathcal{T})}\right)
$$

Proof. First define $\bar{\mu}_{n}: \mathcal{K}\left(D_{n}^{(\mathcal{T})}\right) \rightarrow \mathcal{F}^{\mathcal{T}}$, for any $n \geq 0$ and $d \in \mathcal{K}\left(D_{n}^{(\mathcal{T})}\right)$ by

$$
\bar{\mu}_{n}(d)=\uparrow D \text { where } \mu_{n}(d)=[D]
$$

$\bar{\mu}_{n}$ are monotone by Proposition 26(1). Then the extensions $\tilde{\mu}_{n}: D_{n}^{(\mathcal{T})} \rightarrow \mathcal{F}^{\mathcal{T}}$ of $\bar{\mu}$ defined by

$$
\tilde{\mu}_{n}(x)=\bigsqcup\left\{\bar{\mu}_{n}(d) \mid d \in \mathcal{K}\left(D_{n}^{(\mathcal{T})}\right) \& d \sqsubseteq x\right\}
$$

are continuous. It follows from Proposition 26(2) that for any $n \geq 0, x \in D_{n}^{(\mathcal{T})}$, $\tilde{\mu}_{n}(x)=\tilde{\mu}_{n+1}\left(i_{n}(x)\right)$. Hence, $\mathcal{F}^{\mathcal{T}}$ together with $\tilde{\mu}_{n}$ is a cocone for the $\omega$-chain of $\left(F^{(\mathcal{T})}, D_{0}^{(\mathcal{T})}, i_{0}^{(\mathcal{T})}\right)$. To prove that it is initial, consider another cocone, a domain $E$ with $\sigma_{n}: D_{n}^{(\mathcal{T})} \rightarrow E$ such that $\sigma_{n+1} \circ i_{n}=\sigma_{n}$. We define $\theta: \mathcal{F}^{\mathcal{T}} \rightarrow E$ on compact elements by $\theta(\uparrow A)=\sigma_{n}(a)$ where $a$ is an element of some $\mathcal{K}\left(D_{n}^{(\mathcal{T})}\right)$ such that $\mu_{n}(a)=[A]$. This element exists by Proposition 26(3). We prove that $\theta$ is monotone. Suppose $\uparrow A \subseteq \uparrow B$, i.e. $B \leq A$. By Proposition 26(2)(3) there exist $n \geq 0, a \in \mathcal{K}\left(D_{n}^{(\mathcal{T})}\right)$ and $b \in \mathcal{K}\left(D_{n}^{(\mathcal{T})}\right)$ such that $\mu_{n}(a)=[A]$ and $\mu_{n}(b)=[B]$. It follows from Proposition 26(1) that $a \sqsubseteq b$. Hence $\sigma_{n}(a) \sqsubseteq \sigma_{n}(b)$, since $\sigma_{n}$ are monotone. By the definition of $\theta, \theta(\uparrow A) \sqsubseteq \theta(\uparrow B)$. We have defined $\theta$ to have $\theta\left(\bar{\mu}_{n}(d)\right)=\sigma_{n}(d)$. The continuous extension $\tilde{\theta}: \mathcal{F}^{\mathcal{T}} \rightarrow E$ is the unique mediating morphism such that $\forall n . \tilde{\theta} \circ \tilde{\mu}_{n}=\sigma_{n}$. 


\section{Corollary 28.}

1. If $\mathcal{T}$ is natural split then $\mathcal{F}^{\mathcal{T}} \simeq \mathcal{F}^{\mathcal{B}} \times\left[\mathcal{F}^{\mathcal{T}} \rightarrow \mathcal{F}^{\mathcal{T}}\right]$.

2. If $\mathcal{T}$ is lazy split then $\mathcal{F}^{\mathcal{T}} \simeq \mathcal{F}^{\mathcal{T}} \times\left[\mathcal{F}^{\mathcal{T}} \rightarrow \mathcal{F}^{\mathcal{T}}\right]_{\perp}$.

3. If $\mathcal{T}$ is natural equated then $\mathcal{F}^{\mathcal{T}} \simeq\left[\mathcal{F}^{\mathcal{T}} \rightarrow \mathcal{F}^{\mathcal{T}}\right]$.

4. If $\mathcal{T}$ is lazy equated then $\mathcal{F}^{\mathcal{T}} \simeq\left[\mathcal{F}^{\mathcal{T}} \rightarrow \mathcal{F}^{\mathcal{T}}\right]_{\perp}$.

As expected, we can go in the other direction, proving that a certain kind of colimits could be recovered as filter structures of disciplined intersection type theories.

Definition 29. We define the sets of triples $\mathcal{C}_{i}(i \in\{1,2,3,4\})$ as follows:

$$
\begin{aligned}
& \mathcal{C}_{1}=\left\{\left\langle\operatorname{Hom}^{E},\{\perp\}, \operatorname{tr}\right\rangle \mid E \in \mathbf{A L G}\right\} \\
& \mathcal{C}_{2}=\left\{\left\langle\operatorname{Hom}_{\perp}^{E},\{\perp\}, \operatorname{tr}\right\rangle \mid E \in \mathbf{A L G}\right\} \\
& \mathcal{C}_{3}=\left\{\left\langle\operatorname{Hom}_{E} E, i_{0}\right\rangle \mid E \in \mathbf{A L G} \& i_{0}: E \rightarrow[E \rightarrow E]\right\} \\
& \mathcal{C}_{4}=\left\{\left\langle\operatorname{Hom}_{\perp}, E, i_{0}\right\rangle \mid B \in \mathbf{A L G} \& i_{0}: E \rightarrow[E \rightarrow E]_{\perp}\right\}
\end{aligned}
$$

We define $\mathcal{C}=\mathcal{C}_{1} \cup \mathcal{C}_{2} \cup \mathcal{C}_{3} \cup \mathcal{C}_{4}$.

Theorem 30. Let $\rho=\left(F, D_{0}, i_{0}\right) \in \mathcal{C}$. Then there exists a disciplined intersection type theory $\mathcal{T}$ such that

$$
\operatorname{colim}(\rho) \simeq \mathcal{F}^{\mathcal{T}}
$$

Proof. For each $\rho=\left(F, D_{0}, i_{0}\right) \in \mathcal{C}$, we first define a set $\mathbb{A}^{\rho}$ of atoms.

$$
\mathbb{A}^{\rho}=\{\underline{d} \mid d \in \mathcal{K}(B) \& d \neq \perp\} \cup\{\top\}
$$

Then, we define a preorder $\leq_{\mathcal{B}}$ on $\mathbb{B}\left(\mathbb{A}^{\rho}\right)$ as follows.

$$
\begin{gathered}
\underline{d}_{\underline{\mathcal{B}}} \underline{e} \Leftrightarrow e \sqsubseteq d \\
\underline{d}={ }_{\mathcal{B}} \bigcap_{i=1}^{m} \frac{e_{i}}{\mathrm{~d}} \Leftrightarrow d=\bigsqcup_{i=1}^{m} e_{i} \\
\underline{d} \leq \bar{T}
\end{gathered}
$$

For the cases where the embeddings $i_{0}$ are non-trivial, we define def as follows.

$$
\operatorname{def}(\underline{d})=\bigcap_{i=1}^{m}\left(\underline{a_{i}} \rightarrow \underline{b_{i}}\right) \Leftrightarrow i_{0}(d)=\bigsqcup_{i=1}^{m}\left(a_{i} \Rightarrow b_{i}\right)
$$

It is routine to check that the resulting intersection type theory $\mathcal{T}^{\rho}$ is disciplined in all four cases. Since $B \simeq \mathcal{F}^{\mathcal{B}}$, by Theorem 27 we have that $\mathcal{F}^{\mathcal{T}^{\rho}} \simeq$ $\operatorname{colim}\left(\rho^{\mathcal{T}^{\rho}}\right) \simeq \operatorname{colim}(\rho)$.

We now prove the converse of Corollary 28. These two corollaries together justify the classification shown in Figure 1.

\section{Corollary 31.}

1. If $\mathcal{F}^{\mathcal{T}} \simeq E \times\left[\mathcal{F}^{\mathcal{T}} \rightarrow \mathcal{F}^{\mathcal{T}}\right]$ then $\exists \mathcal{T}^{\prime}$ natural split such that $\mathcal{T} /=\simeq \mathcal{T}^{\prime} /=$.

2. If $\mathcal{F}^{\mathcal{T}} \simeq E \times\left[\mathcal{F}^{\mathcal{T}} \rightarrow \mathcal{F}^{\mathcal{T}}\right]_{\perp}$ then $\exists \mathcal{T}^{\prime}$ lazy split such that $\mathcal{T} \mid=\simeq \mathcal{T}^{\prime} /=$.

3. If $\mathcal{F}^{\mathcal{T}} \simeq\left[\mathcal{F}^{\mathcal{T}} \rightarrow \mathcal{F}^{\mathcal{T}}\right]$ then $\exists \mathcal{T}^{\prime}$ natural equated such that $\mathcal{T} \mid=\simeq \mathcal{T}^{\prime}=$.

4. If $\mathcal{F}^{\mathcal{T}} \simeq\left[\mathcal{F}^{\mathcal{T}} \rightarrow \mathcal{F}^{\mathcal{T}}\right]_{\perp}$ then $\exists \mathcal{T}^{\prime}$ natural equated such that $\mathcal{T} /=\simeq \mathcal{T}^{\prime} /=$.

Proof. We prove only the third case. The rest is similar. Take $\rho=\left(\operatorname{Hom}, \mathcal{F}^{\mathcal{T}}, i_{0}\right)$ where $i_{0}$ is the isomorphism from $D$ to $[D \rightarrow D]$. By the previous theorem, we have that $\mathcal{F}^{\mathcal{T}} \simeq \operatorname{colim}(\rho) \simeq \mathcal{F}^{\mathcal{T}^{\prime}}$ for some $\mathcal{T}^{\prime}$ natural equated. Since $\mathcal{K}\left(\mathcal{F}^{\mathcal{T}}\right) \simeq$ $\mathcal{T} /=$ and $\mathcal{K}\left(\mathcal{F}^{\mathcal{T}^{\prime}}\right) \simeq \mathcal{T}^{\prime} /=$, we conclude that $\mathcal{T} /=\simeq \mathcal{T}^{\prime} /=$. 


\section{References}

1. S. Abramsky and L.C. Ong. Full abstraction in the Lazy Lambda Calculus. Information and Computation, 105: 159-267, 1993.

2. F. Alessi. Strutture di tipi, teoria dei domini, e modelli del $\lambda$-calcolo. PhD Thesis. University of Turin, 1991.

3. F. Alessi, M. Dezani-Ciancaglini and F. Honsell. Inverse Limit Models as Filter Models. In Proceedings of HOR'04, pages 3-25, 2004.

4. H.P. Barendregt The Lambda Calculus: Its syntax and semantics. North-Holland Publishing co., Amsterdam, 1984.

5. H. Barendregt, M. Coppo, and M. Dezani-Ciancaglini. A filter lambda model and the completeness of type assignment. J. Symbolic Logic, 48(4):931-940, 1983.

6. M. Coppo and M. Dezani-Ciancaglini. An extension of the basic functionality theory for the $\lambda$-calculus. Notre Dame J. Formal Logic, 21(4):685-693, 1980.

7. M. Coppo, M. Dezani-Ciancaglini and G. Longo. Applicative information systems. In CAAP '83 (L'Aquila, 1983), pages 35-64. Springer, Berlin,1983.

8. M. Coppo, M. Dezani-Ciancaglini, F. Honsell, and G. Longo. Extended type structures and filter lambda models. In Logic Colloquium '82, pages 241-262. NorthHolland, 1984.

9. M. Coppo, M. Dezani-Ciancaglini, and M. Zacchi. Type theories, normal forms and $D_{\infty}$ lambda models. Information and Compuation, 72(2):85-116, 1987.

10. M. Dezani-Ciancaglini, S. Ghilezan, and S. Likavec. Behavioural inverse limit models. Theoret. Comput. Sci., 316(1-3):49-74, 2004.

11. M.Dezani-Ciancaglini, F.Honsell and F.Alessi. A complete characterization of complete intersection-type preorders. ACM TOCL, 4(1):120-146, 2003.

12. M. Dezani-Ciancaglini, F. Honsell, and Y. Motohama. Compositional characterization of $\lambda$-terms using intersection types. Theoret. Comput. Sci., 304(3):459-495, 2005.

13. G. Gierz, K.H. Hofmann, K. Keimel, D.J. Lawson, M.W. Mislove, and D. Scott. Continuous lattices and domains. Cambridge University Press, 2003.

14. R. Hindley and G. Longo. Lambda calculus models and extensionality. Z. Math Logik Grundlag. Math, 26(4):289-310, 1980.

15. F. Honsell and S. Ronchi Della Rocca. An approximation theorem for topological lambda models and the topological incompleteness of lambda calculus. J. Comput. System Sci., 45(1):49-75, 1992.

16. D. Park. The $Y$-combinator in Scott's $\lambda$-calculus models (revised version). Theory of Computation Report 13, Department of Computer Science, University of Warick, 1976.

17. G. Plotikin. Set-Theoretic and Other Elementary Models of the $\lambda$-Calculus. Theoretical Computer Science, 121: 351-409, 1993.

18. S. Ronchi della Rocca and L. Paolini. The Parametric Lambda Calculus. A Metamodel for Computation. Texts in Theoretical Computer Science. An EACTS Series. Springer-Verlag, 2004.

19. D.S. Scott. Continuous lattices. In Toposes, algebraic geometry and logic, volume 274 of $L N M$, pages 97-136. Springer-Verlag, 1972.

20. Michael B. Smyth and Gordon D. Plotkin. The category-theoretic solution of recursive domain equations. SIAM Journal on Computing, 11(4):761-783, November 1982. 